\title{
Treatment for gastrointestinal and pancreatic neuroendocrine tumours: a network meta-analysis (Protocol)
}

Walter MA, Spanjol M, Kollár A, Bütikofer L, Gloy VL, Dumont RA, Seiler CA, Christ ER, Radojewski P, Briel M, Kaderli RM

Walter MA, Spanjol M, Kollár A, Bütikofer L, Gloy VL, Dumont RA, Seiler CA, Christ ER, Radojewski P, Briel M, Kaderli RM. Treatment for gastrointestinal and pancreatic neuroendocrine tumours: a network meta-analysis (Protocol).

Cochrane Database of Systematic Reviews 2020, Issue 8. Art. No.: CD013700.

DOI: $10.1002 / 14651858 . C D 013700$.

www.cochranelibrary.com

Treatment for gastrointestinal and pancreatic neuroendocrine tumours: a network meta-analysis (Protocol) Copyright $\odot 2020$ The Cochrane Collaboration. Published by John Wiley \& Sons, Ltd. 
TABLE OF CONTENTS

HEADER 1

ABSTRACT

BACKGROUND

OBJECTIVES

METHODS

ACKNOWLEDGEMENTS

REFERENCES

APPENDICES

WHAT'S NEW

HISTORY

CONTRIBUTIONS OF AUTHORS

DECLARATIONS OF INTEREST

SOURCES OF SUPPORT 
[Intervention Protocol]

\section{Treatment for gastrointestinal and pancreatic neuroendocrine tumours: a network meta-analysis}

Martin A Walter ${ }^{1}$, Marko Spanjol ${ }^{1}$, Attila Kollár ${ }^{2}$, Lukas Bütikofer ${ }^{3}$, Viktoria L Gloy ${ }^{4}$, Rebecca A Dumont ${ }^{1}$, Christian A Seiler ${ }^{5}$, Emanuel R Christ $^{6}$, Piotr Radojewski ${ }^{1}$, Matthias Briel ${ }^{4}$, Reto M Kaderli ${ }^{5}$

${ }^{1}$ Nuclear Medicine Division, Diagnostic Department, University Hospitals Geneva (HUG), Geneva, Switzerland. ${ }^{2}$ Department of Medical Oncology, Bern University Hospital, University of Bern, Bern, Switzerland. ${ }^{3}$ Clinical Trials Unit, Bern, University of Bern, Bern, Switzerland. ${ }^{4}$ Department of Clinical Research, University Hospital Basel and University of Basel, Basel, Switzerland. ${ }^{5}$ Department of Visceral Surgery and Medicine, Bern University Hospital, University of Bern, Bern, Switzerland. ${ }^{6}$ Department of Endocrinology, Diabetes, and Metabolism, Basel University Hospital, University of Basel, Basel, Switzerland

Contact address: Martin A Walter, Martin.Walter@unige.ch.

Editorial group: Cochrane Gynaecological, Neuro-oncology and Orphan Cancer Group.

Publication status and date: New, published in Issue 8, 2020.

Citation: Walter MA, Spanjol M, Kollár A, Bütikofer L, Gloy VL, Dumont RA, Seiler CA, Christ ER, Radojewski P, Briel M, Kaderli RM. Treatment for gastrointestinal and pancreatic neuroendocrine tumours: a network meta-analysis (Protocol). Cochrane Database of Systematic Reviews 2020, Issue 8. Art. No.: CD013700. DOI: 10.1002/14651858.CD013700.

Copyright @ 2020 The Cochrane Collaboration. Published by John Wiley \& Sons, Ltd.

\section{A B S T R A C T}

\section{Objectives}

This is a protocol for a Cochrane Review (intervention). The objectives are as follows:

To evaluate the safety and efficacy of therapies for neuroendocrine tumours (NETs), to guide clinical decision-making, and to provide estimates of relative efficiency of the different treatment options (including placebo) and rank the treatments according to their efficiency. 


\section{B A C K G R O U N D}

\section{Description of the condition}

Neuroendocrine tumours (NETs), sometimes referred to as carcinoid tumours, are a heterogenous group of malignancies (cancers) that arise from cells of the endocrine (hormonal) and neurological systems. They have an estimated overall 20year duration prevalence of 171,321 and a yearly age-adjusted incidence of 6.98 cases per 100,000 according to the National Cancer Institute's Surveillance, Epidemiology, and End Results (SEER) 18 registry (Dasari 2017). A population-based study found a 6.4 fold increase in incidence between 1973 and 2012 (Dasari 2017). NETs are more common with higher age with an incidence among patients 65 years or older of 25 per 100,000 . About $61.0 \%$ of NETs derive from the gastrointestinal tract or the pancreas (Lawrence 2011), and accordingly these tumours are called gastroenteropancreatic NET (GEP-NET). Other sites for primary NET include lungs, thyroid, ovaries, cervix, pituitary, and adrenal glands (Hallet 2015).

The relative frequency and annual incidence rate per 100,000 of GEP-NETs differ site by site and, in some cases, change over time and are different between countries and continents (Fraenkel 2014). NETs of the rectum are the most common in east Asia and the USA, while small intestinal NETs are the most common in males, and appendiceal NETs the most common in females in the UK (Fraenkel 2012; Fraenkel 2014). Racial discrepancies have been found in the US SEER registry, with small intestinal NETS being more often in African Americans than in the White population (DePalo 2019).

Most GEP-NET are sporadic, but approximately 5\% arise in the context of cancer predisposition syndromes (Clift 2020). Neuroendocrine tumours, especially those of the pancreas (pNET), may be associated with familial syndromes. Multiple endocrine neoplasia type 1 (MEN 1 ) is the most common familial syndrome associated with NET, while Von-Hippel Lindau syndrome, neurofibromatosis type- 1 and tuberous sclerosis are rarer.

Depending on localisation and stage of the disease, they present with a broad clinical spectrum, from asymptomatic patients with an incidental discovery on imaging to florid endocrinopathy. Up to $30 \%$ to $40 \%$ of GEP-NETs may be secretory (i.e. "functional"), releasing a variety of hormones and hormone-like substances (Clift 2020). Serotonin-secreting small bowel NETs may lead to cardiac valve fibrosis (carcinoid heart disease) as a consequence of hormone hyper-secretion.

The diagnosis of GEP-NETs is usually based on a histopathology that demonstrates neuroendocrine features, such as positive immunohistochemical staining for synaptophysin and chromogranin A. The grading of GEP-NETs, on the other hand, is based on the mitotic index using $\mathrm{Ki}-67$ immunohistochemistry. The World Health Organization (WHO) classification divides NETs according to their proliferative activity into grade 1 (Ki-67 index $\leq 2 \%$ ) and grade 2 (Ki-67 index $3 \%$ to $20 \%$ ). Based on their morphological characteristics, grade 3 tumours are subdivided into well-differentiated NET and poorly-differentiated neuroendocrine carcinomas, both with Ki-67 index $>20 \%$ (Klimstra 2019). The grading aids in the prognostication of survival: The five-year survival rates of grade 1,2 and 3 NETs are $96 \%, 73 \%$ and
$28 \%$, respectively (Ramage 2012). Grade 1 NETs have the highest incidence with 2.53 per 100,000 (Dasari 2017).

\section{Description of the intervention}

Tumour growth, treatment and outcome vary considerably with the location of the primary lesions, as well as with their grade, extension, and stage (Lawrence 2011; Modlin 2008; Yao 2008). A broad spectrum of therapeutic options permits staged disease management with various treatment combinations and sequencing. This approach, however, requires a highly interdisciplinary and dynamic approach, which typically involves physicians of various specialties who work in concert to manage these often-complex cases and select a treatment strategy from an array of available options.

Management strategies depend on primary tumour, locoregional and distant metastases, tumour-related symptoms, syndromes and presence of carcinoid heart disease. Depending on primary tumour size and site, NETs are treated surgically whenever feasible, as this is the only potentially curative treatment (Yao 2008). In metastatic, well-differentiated NETs, somatostatin analogues (SSA), and interferon alpha (IFN) as a possible second-line therapy, are a cornerstone in the palliative setting, as effective means of improving quality of life (QoL) and delaying disease progression (Cives 2014; Clift 2020). More recently, molecularly targeted drugs like the mTOR-inhibitor everolimus, the multi-targeted receptor tyrosine kinase inhibitor sunitinib, and the vascular endothelial growth factor (VEGF) antibody bevacizumab have been introduced into the clinical setting following trials demonstrating efficacy in people with progressive NET (Kunz 2013; Pavel 2016; Yao 2017). The radiolabelled somatostatin receptor ligand lutetium-177DOTATATE also recently demonstrated a benefit over treatment with somatostatin analogues alone in people with progressive NET (Strosberg 2017). Liver-directed therapies further broaden the therapeutic landscape (Pavel 2016). In advanced grade 3 pNET and advanced symptomatic or progressive grade 1 or 2 pNET, systemic chemotherapy with streptozocin- or temozolomide-based regimens is the first choice of treatment. In grade 3 NEC, platinumbased chemotherapy is recommended as a first-line therapy (Pavel 2016).

\section{Why it is important to do this review}

Several available therapies have demonstrated efficacy in randomised controlled trials (RCTs). However, translation of these results into improved care faces several challenges, as several therapies were compared with placebo only and a direct comparison of the most pertinent therapies is incomplete (Kaderli 2019). In a previous systematic review and network meta-analysis on pNETs and neuroendocrine tumours of the gastrointestinal tract (GI-NETs), we found several monotherapies that were superior to placebo, including everolimus, interferon, and sunitinib in PNETs and somatostatin analogues in pNETs and GI-NETs (Kaderli 2019). Furthermore, the results suggested a superiority of combination therapies, especially those including somatostatin analogues. On the other hand, NET therapies have a broad range of risk for adverse events and effects on QoL, which need to be considered while choosing the appropriate treatment. A systematic comparison of benefits and harms of all currently available therapeutic modalities will allow informed clinical decision-making for clinicians, patients and policy makers. 
Furthermore, there is ongoing research in the treatment of NETs. Surufatinib has demonstrated a higher progression-free survival in GI-NETs in the SANET-ep trial (NCT02588170; Xu 2019). New results for axitinib and somatostatin analogue are expected in GI-NET (AXINET trial, NCT01744249), for surufatinib, everolimus and streptozocin plus fluorouracil in pNET (SANET-p, NCT02589821 and SEQTOR trial, NCT02246127), and for lutetium-177 (177Lu)DOTATATE and everolimus both in GI-NET and PNET (COMPETE trial, NCT03049189). It is therefore vital to provide a regularly updated systematic review and network meta-analysis for clinical decision-making based on the best available and most recent evidence.

\section{OB JECTIVES}

To evaluate the safety and efficacy of therapies for neuroendocrine tumours (NETs), to guide clinical decision-making, and to provide estimates of relative efficiency of the different treatment options (including placebo) and rank the treatments according to their efficiency.

\section{ME T HOD S}

\section{Criteria for considering studies for this review \\ Types of studies}

We will include randomised controlled trials (RCTs), including randomised controlled cross-over trials.

\section{Types of participants}

People of any age with any type and any stage GEP-NETs (gastroenteropancreatic NETs).

\section{Types of interventions}

We will include RCTs comparing at least two treatments of any kind (including usual care or placebo) in NETs, administered in any way.

Examples of treatments include the mechanistic target of rapamycin inhibitor everolimus (Yao 2016), the multi-targeted receptor tyrosine kinase inhibitor sunitinib (Raymond 2011), the vascular endothelial growth factor (VEGF) antibody bevacizumab (Yao 2017), the radiolabelled somatostatin analogue lutetium-177 (177Lu)-dotatate (Strosberg 2017), and new combinations of previously established therapies (Pavel 2011). Several therapies will be compared only with placebo, while others will be directly compared.

\section{Types of outcome measures}

\section{Primary outcomes}

1. Disease control after 12 months

2. Progression-free survival

\section{Secondary outcomes}

1. Overall survival

2. Occurrence of adverse events according to the treatment applied (grades 3 to 4 , any grade)

3. Quality of life (QoL)

Disease control is defined as the sum of complete response, partial response and stable disease, or as the total minus the number disease progressions. We will apply a continuity correction for studies with a zero cell count by adding 0.5 to all cell frequencies. Adverse events will be classified according to the National Cancer Institute Common Terminology Criteria for Adverse Events ( $\mathrm{NCl}$ 2010): Grade 1 corresponds to mild, grade 2 to moderate, grade 3 to severe or medically significant, and grade 4 to life-threatening adverse events. Effects on QoL will be quantified based on the QoL Questionnaire C3O of the European Organization for Research and Treatment of Cancer (EORTC QLQ-30).

\section{Search methods for identification of studies}

\section{Electronic searches}

We will identify trials through systematic searches of the following bibliographic databases:

- Cochrane Central Register of Controlled Trials (CENTRAL) in the Cochrane Library

- MEDLINE (Ovid)

- Embase (Ovid)

In addition, we will check trial registries (ClinicalTrials.gov and the World Health Organization International Clinical Trials Registry Platform Search Portal [apps.who.int/trialsearch/]) for ongoing or unpublished eligible trials and manually search for abstracts from scientific and clinical meetings related to NETs in 2019 and 2020 (annual ENETS conference and neuroendocrine tumour symposium of the NANETS).

We will search all databases from 1 January 1947, until present, and we will impose no restriction on language of publication. Appendix 1; Appendix 2; Appendix 3.

\section{Searching other resources}

We will scan the reference lists of the included RCT reports and relevant review articles for additional references.

\section{Data collection and analysis}

\section{Selection of studies}

With two review authors working in duplicate, we will screen all abstracts and obtain the full-text report of potentially relevant studies. Subsequently, we will screen all potentially relevant studies in the same way. Any discordance will be resolved by a third review author.

\section{Data extraction and management}

We will use a data collection form for study characteristics and outcome data which has been piloted in our previous systematic review and network meta-analysis on therapeutic options for neuroendocrine tumours (Kaderli 2019). One of the review authors will extract study characteristics from included studies, and a second review author will verify the extractions. We will extract the following study characteristics.

1. Characteristics of included trials: first author, year of publication, study origin, type of treatments, median duration and median follow-up of each treatment, percentage of people with complete follow-up, availability of a sample size calculation, and number of participants randomised for each treatment. 
2. Participant data: separately for each treatment: primary tumour site, tumour grading, presence of metastases and functional tumours, percentage of female participants and the participants' median/mean age; main primary tumour (pNET and/or GI-NET) for all treatments.

3. Clinical outcomes: complete response, partial response, stable disease, disease control, disease progression, progression-free survival, overall survival, occurrence of adverse events (grade 3 to 4 , any grade), and QoL.

Any discordance will be resolved by a third review author. Data will be entered into Review Manager software (RevMan 2014) and checked by a second review author for accuracy.

\section{Assessment of risk of bias in included studies}

Two review authors will independently assess the risk of bias for each RCT, using the Cochrane 'Risk of bias' tool (Higgins 2019), which utilises the following domains.

1. Random sequence generation

2. Allocation concealment

3. Blinding of participants and personnel

4. Blinding of outcome assessment

5. Completeness of outcome data

6. Selectivity of reporting

7. Other bias (including baseline imbalance, protocol deviations, inappropriate influence of funders)

We will provide a summary 'Risk of bias' assessment for each study using the method outlined in the Cochrane Handbook for Systematic Reviews of Interventions (Higgins 2019). Each domain will be rated as low (bias is unlikely to seriously alter the results), high (bias is likely to seriously weaken confidence in results), or unclear risk of bias. All discordance will be resolved by a third review author.

\section{Measures of treatment effect}

We will use odds ratios as effect measure for disease control after 12 months and hazard ratios as effect measure for progressionfree survival, both accompanied by $95 \%$ confidence intervals (95\% Cls). We will summarise all results using forest plots with combined effect estimates and size of squares proportional to the inverse of the standard errors. We will present secondary outcomes for each intervention (if available) using descriptive statistics-i.e. number and percentage of adverse events, and mean and standard deviation of the change of QoL.

We will rank treatments based on $P$ values, measuring the extent of certainty that a treatment is better than another one, averaged over all competing treatments (Rücker 2015).

\section{Unit of analysis issues}

The analysis will be made at the individual allocation level. For cross-over trials, we will use data from paired analyses when available. In cross-over studies where paired analyses are not reported, we will use data from the first trial period if they are presented separately. We will exclude cross-over studies from the analysis if only data for the first and second periods combined are available.
Multi-arm trials will be included in the network meta-analysis. The correlation of treatment effects on different comparisons will be accounted for by re-weighting all comparisons of each multi-arm study (Rücker 2012; Rücker 2014).

\section{Dealing with missing data}

We will contact authors of included RCT reports for information on unreported outcomes and missing outcome data in their studies.

If a RCT report does not report hazard ratios and further data cannot be obtained by contacting authors, we will estimate the hazard ratios from reconstructed Kaplan-Meier curves (if available) by using a Cox-proportional hazard model.

\section{Assessment of heterogeneity}

We will assess heterogeneity using all pairwise comparisons available from more than one trial. We will calculate the betweenstudy variance $\mathrm{T}^{2}$, the within design component of Cochran's $Q$ (i.e. the weighted sum of squared differences between pairwise comparisons from multiple trials) and the associated $\mathrm{I}^{2}$ (percentage of variation across studies due to heterogeneity rather than chance). If quantification of heterogeneity is not possible (i.e. if there is no comparison done in more than one trial), we will fit fixedeffect models; otherwise, we will use random-effects models.

We will assess inconsistency using closed loops within the network (if any) and calculate the between design component of Cochran's $\mathrm{Q}$ and the associated $\mathrm{I}^{2}$. In addition, we will perform a netsplit analysis and compare direct and indirect estimates via a ratio of odds or hazard ratios.

We will calculate the total Cochrane's $Q$ as the sum of between and within designs component and the associated $\mathrm{I}^{2}$.

Inconsistency and intransitivity will also be assessed qualitatively using the Grading of Recommendations Assessment, Development, and Evaluation (GRADE) approach (Brignardello-Petersen 2018; Puhan 2014; Salanti 2014, see below for more details).

\section{Assessment of reporting biases}

To assess the risk for reporting bias we will first search for a protocol for each of the included studies. For this we will go through the reference lists of corresponding published articles. If there is no reference to a protocol, we will search PubMed, Embase, and the internet for a protocol. If a protocol is available, we will compare the mentioned outcomes and planned statistical analyses in the protocol with those in the published report. If no protocol is available, we will use information from a corresponding registry entry of the included study to compare planned outcomes and analyses with those in the published report. If neither a protocol, nor a registry entry is available, we will compare the outcomes and described analyses in the methods section of the published report with those reported in the results section. Any unexplained differences between the protocol, registry entry, or methods section and the reported results provide evidence for an increased risk of reporting bias of an included study.

If there are 10 or more included studies for individual pairwise meta-analyses, we will create funnel plots for visual inspection to detect potential asymmetry. 


\section{Data synthesis}

We will separately analyse two different outcomes (disease control and progression-free survival) and two types of NET (PNET and GI-NET) in four network meta-analyses. The NET types will be distinguished to ensure that the selected studies are similar except for the interventions being compared. If a study includes several NET types, we will include the respective subgroup analyses (if available). Otherwise we will rely on expert opinion whether or not to include the study and will use sensitivity analyses to assess the effect of the decision.

Before including an intervention in the network meta-analysis, we will assess the respective study populations critically in terms of the transitivity assumption. Interventions only given to a subset of participants (i.e. those critically ill) will not be included or excluded in a sensitivity analysis. However, since the network is currently very sparse, the benefit of additional studies might outweigh a certain risk of violation of the transitivity assumption. The comparison among all interventions (including placebo) are of interest and we will not define a decision and a supplementary set. However, if more data become available, we might focus on a specific set of interventions.

Because the network is sparse, we will merge similar interventions, i.e. different doses, administration intervals and routes of application of the same compound. When more data become available, we will consider splitting nodes if the effects are suspected to be different.

We will perform the network meta-analyses with a frequentist approach using R-package (R Core 2019) netmeta (Rücker 2020), and/or the Review Manager Web (RevMan Web 2019, if applicable). If quantification of heterogeneity is possible, i.e. if there are pairwise comparisons included in more than one trial, we will use random-effects models. Otherwise, we will use fixed-effect models. Validity of the network in terms of consistency will be assessed quantitatively by comparing direct and indirect estimates for each loop of the network and qualitatively using GRADE (as described in section Assessment of heterogeneity).

\section{Subgroup analysis and investigation of heterogeneity}

In view of the small number of likely RCTs to be included in this review, we will refrain from any subgroup analysis, including subgroup analysis based on tumour grading, since the separate analysis for each treatment included in a RCT is frequently missing.

If applicable, we will assess heterogeneity by the between-studyvariance $\mathrm{T}^{2}$, Cochran's $\mathrm{Q}$, and $\mathrm{I}^{2}$. If applicable, we will quantify inconsistency by a netsplit analysis, in which direct and indirect estimates will be compared via a ratio of odds or hazard ratios. We will also calculate the between-design part of Cochran's $Q$.

If there is evidence for heterogeneity, we will assess participant and trial characteristics for a potential source of the heterogeneity.

\section{Sensitivity analysis}

Currently, the network is very sparse and we will not be able to undertake sensitivity analyses. If sufficient trials are identified, we will consider several sensitivity analyses for the primary outcomes. We will for example, only use low risk of bias trials, exclude trials with a mixture of different types of NETs and use alternative or no merging of nodes. We will also consider different analytical approaches, such as fixed-effect only, or a Bayesian instead of the specified frequentist approach (e.g. using R package BUGSnet (Béliveau 2019)).

\section{Summary of findings and assessment of the certainty of the evidence}

We will use the GRADE approach to assess confidence in estimates of effect (certainty of evidence) associated with specific comparisons, including estimates from direct, indirect, and final network meta-analysis (Brignardello-Petersen 2018; Puhan 2014; Salanti 2014). Our confidence assessment will address risk of bias (limitations in study design and execution), inconsistency (heterogeneity of estimates of effects across trials), indirectness (differences in population, interventions, or outcomes to the target of the network meta-analysis) and imprecision (e.g. 95\% confidence intervals are wide and include or are close to null effect). Limitations in any of these domains will result in a decrease of the certainty of evidence from high to moderate, low, or very low certainty by -1 (serious concern) or -2 (very serious concern). We will base indirect evidence on the most dominant loops (i.e. the shortest path between two treatments) and potentially rate it down for intransitivity (differences in study characteristics that may modify treatment effect in the direct comparisons along the path). We will obtain the final network meta-analysis confidence rating from the higher of the direct and indirect rating excluding imprecision and we will rate it down for imprecision and incoherence (difference between direct and indirect estimates).

\section{ACKNOWLEDGEMENTS}

We would like to thank the following members of Cochrane Gynaecological, Neuro-oncology and Orphan Cancers (GNOC); Jo Morrison (Co-ordinating Editor) for clinical advice, Gail Quinn (Managing Editor) and Tracey Harrison (Assistant Manging Editor) for editorial assistance.

Cochrane Switzerland will assist with the electronic libraries search.

This project was supported by the National Institute for Health Research (NIHR), via Cochrane infrastructure funding to the Cochrane Gynaecological, Neuro-oncology and Orphan Cancers Group. The views and opinions expressed therein are those of the review authors and do not necessarily reflect those of the Systematic Reviews Programme, NIHR, NHS, or the Department of Health.

The authors and GNOC, are grateful to the following peer reviewers for their time and comments: Martyn Caplin, Emma Cattell, Alysia De Nino and Julie Walther. 


\section{RE F E R E N C E S}

\section{Additional references}

\section{Brignardello-Petersen 2018}

Brignardello-Petersen R, Bonner A, Alexander PE, Siemieniuk RA, Furukawa TA, Rochwerg B, et al, GRADE Working Group. Advances in the GRADE approach to rate the certainty in estimates from a network meta-analysis. Journal of Clinical Epidemiology 2018;93:36-44.

\section{Béliveau 2019}

Béliveau A, Boyne DJ, Slater J, Brenner D, Arora P. BUGSnet: an $R$ package to facilitate the conduct and reporting of Bayesian network meta-analyses. BMC Medical Research Methodology 2019;19(1):196.

\section{Cives 2014}

Cives M, Strosberg J. An update on gastroenteropancreatic neuroendocrine tumors. Oncology (Williston Park) 2014;28(9):749-56, 758

\section{Clift 2020}

Clift AK, Kidd M, Bodei L, Toumpanakis C, Baum RP, Oberg K, et al. Neuroendocrine neoplasms of the small bowel and pancreas. Neuroendocrinology 2020;110(6):444-76.

\section{Dasari 2017}

Dasari A, Shen C, Halperin D, Zhao B, Zhou S, Xu Y, et al. Trends in the incidence, prevalence, and survival outcomes in patients with neuroendocrine tumors in the United States. JAMA Oncology 2017;3(10):1335-42.

\section{DePalo 2019}

DePalo DK, Lee RM, Lopez-Aguiar AG, Gamboa AC, Rocha F, Poultsides $\mathrm{G}$, et al, other members of the United States Neuroendocrine Tumor Study Group. Interaction of race and pathology for neuroendocrine tumors: epidemiology, natural history, or racial disparity? Journal of Surgical Oncology 2019;120(6):919-25.

\section{Fraenkel 2012}

Fraenkel M, Kim MK, Faggiano A, Valk GD. Epidemiology of gastroenteropancreatic neuroendocrine tumours. Best Practice \& Research Clinical Gastroenterology 2012;26(6):691-703.

\section{Fraenkel 2014}

Fraenkel M, Kim M, Faggiano A, de Herder WW, Valk GD, Knowledge NETwork. Incidence of gastroenteropancreatic neuroendocrine tumours: a systematic review of the literature. Endocrine Related Cancer 2014;21(3):R153-63.

\section{Hallet 2015}

Hallet J, Law CH, Cukier M, Saskin R, Liu N, Singh S. Exploring the rising incidence of neuroendocrine tumors: a populationbased analysis of epidemiology, metastatic presentation, and outcomes. Cancer 2015;121(4):589-97.

\section{Higgins 2019}

Higgins JP, Thomas J, Chandler J, Cumpston M, Li T, Page MJ, et al. Cochrane Handbook for Systematic Reviews of Interventions version 6.0 (updated July 2019). The Cochrane Collaboration, 2019. Available from www.training.cochrane.org/handbook.

\section{Kaderli 2019}

Kaderli RM, Spanjol M, Kollár A, Bütikofer L, Gloy V, Dumont RA, et al. Therapeutic options for neuroendocrine tumors: a systematic review and network meta-analysis. JAMA Oncology 2019;5(4):480-9.

\section{Klimstra 2019}

Klimstra DS, Kloppell G, La Rosa S, Rindi G. Classification of neuroendocrine neoplasms of the digestive system. In: WHO Classification of Tumours Editorial Board, editors(s). WHO Classification of Tumours: Digestive System Tumours. 5th edition. Lyon: International Agency for Research on Cancer, 2019:16.

\section{Kunz 2013}

Kunz PL, Reidy-Lagunes D, Anthony LB, Bertino EM, Brendtro K, Chan JA, el al, North American Neuroendocrine Tumor Society. Consensus guidelines for the management and treatment of neuroendocrine tumors. Pancreas 2013;42(4):557-77.

\section{Lawrence 2011}

Lawrence B, Gustafsson BI, Chan A, Svejda B, Kidd M, Modlin IM. The epidemiology of gastroenteropancreatic neuroendocrine tumors. Endocrinology and Metabolism Clinics of North America 2011;40(1):1-18.

\section{Modlin 2008}

Modlin IM, Oberg K, Chung DC, Jensen RT, de Herder WW, Thakker RV, et al. Gastroenteropancreatic neuroendocrine tumours. Lancet Oncology 2008;9(1):61-72.

\section{$\mathrm{NCl} 2010$}

National Cancer Institute. Common Terminology Criteria for Adverse Events (CTCAE) Version 4.03. https://evs.nci.nih.gov/ftp1/CTCAE/CTCAE_4.03/ CTCAE_4.03_2010-06-14_QuickReference_5x7.pdf

\section{Pavel 2011}

Pavel ME, Hainsworth JD, Baudin E, Peeters M, Hörsch D, Winkler RE, et al, RADIANT-2 Study Group. Everolimus plus octreotide long-acting repeatable for the treatment of advanced neuroendocrine tumours associated with carcinoid syndrome (RADIANT-2): a randomised, placebo-controlled, phase 3 study. Lancet 2011;378(9808):2005-12.

\section{Pavel 2016}

Pavel M, O'Toole D, Costa F, Capdevila J, Gross D, Kianmanesh R, et al, Vienna Consensus Conference participants. ENETS Consensus Guidelines Update for the management of distant metastatic disease of intestinal, pancreatic, bronchial Neuroendocrine Neoplasms (NEN) and NEN of unknown primary site. Neuroendocrinology 2016;103(2):172-85.

\section{Puhan 2014}

Puhan MA, Schünemann HJ, Murad MH, Li T, BrignardelloPetersen R, Singh JA, et al, GRADE Working Group. A 
GRADE Working Group approach for rating the quality of treatment effect estimates from network meta-analysis. $B M J$ 2014;349:g5630.

\section{R Core 2019 [Computer program]}

R Foundation for Statistical Computing R: A Language and Environment for Statistical Computing. R Core Team. Vienna, Austria: R Foundation for Statistical Computing, 2019. https:// www.R-project.org/.

\section{Ramage 2012}

Ramage JK, Ahmed A, Ardill J, Bax N, Breen DJ, Caplin ME, et al, UK and Ireland Neuroendocrine Tumour Society. Guidelines for the management of gastroenteropancreatic neuroendocrine (including carcinoid) tumours (NETs). Gut 2012;61(1):6-32.

\section{Raymond 2011}

Raymond E, Dahan L, Raoul JL, Bang YJ, Borbath I, LombardBohas $C$, et al. Sunitinib malate for the treatment of pancreatic neuroendocrine tumors. New England Journal of Medicine 2011;364(11):1082.

\section{RevMan 2014 [Computer program]}

Nordic Cochrane Centre, The Cochrane Collaboration Review Manager (RevMan). Version 5.3. Copenhagen: Nordic Cochrane Centre, The Cochrane Collaboration, 2014.

\section{RevMan Web 2019 [Computer program]}

The Cochrane Collaboration Review Manager Web (RevMan Web). The Cochrane Collaboration, 2019. Available at revman.cochrane.org.

\section{Rücker 2012}

Rücker G. Network meta-analysis, electrical networks and graph theory. Research Synthesis Methods 2012;3(4):312-24.

\section{Rücker 2014}

Rücker G, Schwarzer G. Reduce dimension or reduce weights? Comparing two approaches to multi-arm studies in network meta-analysis. Statistics in Medicine 2014;33(25):4353-69.

\section{Rücker 2015}

Rücker G, Schwarzer G. Ranking treatments in frequentist network meta-analysis works without resampling methods. BMC Medical Research Methodology 2015;15:58.

\section{Rücker 2020}

Rücker G, Krahn U, König J, Efthimiou O, Schwarzer G. Netmeta: Network Meta-Analysis using Frequentist Methods. R package 2020.

\section{Salanti 2014}

Salanti G, Del Giovane C, Chaimani A, Caldwell DM, Higgins JP. Evaluating the quality of evidence from a network metaanalysis. PLOS One 2014;9(7):e99682.

\section{Strosberg 2017}

Strosberg J, El-Haddad G, Wolin E, Hendifar A, Yao J, Chasen B, et al, NETTER-1 Trial Investigators. Phase 3 Trial of 177 LuDotatate for midgut neuroendocrine tumors. New England Journal of Medicine 2017;376(2):125-35.

\section{Xu 2019}

Xu J, Shen L, Zhou Z, Li J, Bai C, Chi Y, et al. Efficacy and safety of surufatinib in patients with well-differentiated advanced extrapancreatic neuroendocrine tumors: results from the randomized phase III study (SANET-ep). Annals of Oncology 2019;30 (suppl_5):v851-v934.

\section{Yao 2008}

Yao JC, Hassan M, Phan A, Dagohoy C, Leary C, Mares JE, et al. One hundred years after "carcinoid": epidemiology of and prognostic factors for neuroendocrine tumors in 35,825 cases in the United States. Journal of Clinical Oncology 2008;26(18):3063-72.

\section{Yao 2016}

Yao JC, Fazio N, Singh S, Buzzoni R, Carnaghi C, Wolin E, et al, RAD001 in Advanced Neuroendocrine Tumours, Fourth Trial (RADIANT-4) Study Group. Everolimus for the treatment of advanced, non-functional neuroendocrine tumours of the lung or gastrointestinal tract (RADIANT-4): a randomised, placebocontrolled, phase 3 study. Lancet 2016;387(10022):968-77.

\section{Yao 2017}

Yao JC, Guthrie KA, Moran C, Strosberg JR, Kulke MH, Chan JA, et al. Phase III prospective randomized comparison trial of depot octreotide plus interferon alfa- $2 b$ versus depot octreotide plus bevacizumab in patients with advanced carcinoid tumors: SWOG S0518. Journal of Clinical Oncology 2017;35(15):1695-703.

\section{APPENDICES}

\section{Appendix 1. Search strategy for the Cochrane Central Register of Controlled Trials}

\begin{abstract}
([mh^"Neuroendocrine tumours"] or [mh "Adenoma, Acidophil"] or [mh "Adenoma, Basophil"] or [mh "Adenoma, Chromophobe"] or [mh Apudoma] or [mh "Carcinoid tumour"] or [mh "Malignant Carcinoid Syndrome"] or [mh "Carcinoma, Neuroendocrine"] or [mh "Carcinoma, Medullary"] or [mh "Carcinoma, Merkel Cell"] or [mh Somatostatinoma] or [mh Vipoma] or [mh Neurilemmoma] or [mh Paraganglioma]) and [mh "Gastrointestinal Neoplasms"]) OR (((Gastroenteropancreatic or Gastro-enteric pancreatic or Gastro-entero-pancreatic or pancreas or pancreatic) and (neuroendocrine and (tumour ${ }^{\star}$ or tumour ${ }^{\star}$ or neoplasm ${ }^{\star}$ or carcinoma*))) or GEPNET* or GEP-NET* or GEPNEC* or GEP-NEC*
\end{abstract}


(Continued)

Therapy search filter therapy or "diet therapy" or "drug therapy" or radiotherapy or surgery or segmentectomy or resection or debulk* or cryoablat* or cryosurger* or radioablat* or radiofrequency ablat* or radio-frequency ablat* or RFablat* or thermoablat* or Cryosurgery or Hepatectomy or "Liver transplant*" or "local ablat" or "transarterial embolization" or "transarterial embolisation" or "transarterial chemoembolization" or "transarterial chemoembolisation" or radioembolization or radioembolisation or somatostatin or chemotherapy or chemotherapies or "peptide receptor radiotherapy" or "targeted molecular therapy" or radiopeptide or DOTATOC or DOTATATE or PRRT

\section{Appendix 2. Search strategy for MEDLINE (Ovid)}

("Neuroendocrine tumours"[Mesh:NoExp] OR "Adenoma, Acidophil"[Mesh] OR "Adenoma, Basophil"[Mesh] OR "Adenoma, Chromophobe"[Mesh] OR "Apudoma"[Mesh] OR "Carcinoid tumour"[Mesh] OR "Malignant Carcinoid Syndrome"[Mesh] OR "Carcinoma, Neuroendocrine"[Mesh] OR "Carcinoma, Medullary"[Mesh] OR "Carcinoma, Merkel Cell"[Mesh] OR "Somatostatinoma"[Mesh] OR "Vipoma"[Mesh] OR "Neurilemmoma"[Mesh] OR "Paraganglioma"[Mesh]) AND "Gastrointestinal Neoplasms"[Mesh]) OR ("Pancreatic Neoplasms"[Mesh:NoExp] AND neuroendocrine[tiab]) OR "Adenoma, Islet Cell"[Mesh] OR "Insulinoma"[Mesh] OR "Carcinoma, Islet CelI"[Mesh] OR "Gastrinoma"[Mesh] OR "Glucagonoma"[Mesh] OR ((gastroenteropancreatic OR gastro-enteric pancreatic OR gastro-entero-pancreatic OR pancreas OR pancreatic) AND (neuroendocrine AND (tumour OR tumours OR tumour OR tumours OR neoplasm OR neoplasms OR carcinoma OR carcinomas)) OR GEPNET` OR GEP-NET* OR GEPNEC* OR GEP-NEC*

Therapy search filter therapy[sh] OR "diet therapy"[sh] OR "drug therapy"[sh] OR radiotherapy[sh] OR surgery[sh] OR segmentectomy OR resection OR debulk* OR cryoablat* OR cryosurger* OR radioablat* OR radiofrequency ablat* OR radio-frequency ablat* OR RFablat* OR thermoablat* OR "Cryosurgery"[Mesh] OR "Hepatectomy"[MeSH] OR Liver transplant OR local ablat* OR transarterial embolization OR transarterial embolisation OR transarterial chemoembolization OR transarterial chemoembolisation $\mathrm{OR}$ radioembolization $\mathrm{OR}$ radioembolisation OR somatostatin OR chemotherapy OR chemotherapies OR peptide receptor radiotherapy OR targeted molecular therapy OR radiopeptide OR DOTATOC OR DOTATATE OR PRRT

Study design filter randomized controlled trial[pt] OR controlled clinical trial[pt] OR randomized[tiab] OR placebo[tiab] OR "drug therapy"[sh] OR randomly[tiab] OR trial[tiab] OR groups[tiab]) NOT ("animal$\mathrm{s}$ "[mh] NOT ("humans"[mh] AND "animals"[mh])

Appendix 3. Search strategy for Embase (Ovid)

((Neuroendocrine tumour/ or (adenoma adj3 acidophil ${ }^{\star}$ ).ti,ab. or (adenoma adj3 basophil).ti,ab. or Chromophobe adenoma/ or Apudoma/ or Carcinoid/ or Carcinoid syndrome/ or (carcinoma adj3 neuroendocrine).ti,ab. or Medullary carcinoma/ or Merkel cell tumour/ or Somatostatinoma/ or Vipoma/ or Neurilemoma/ or Paraganglioma/) and (Gastrointestinal tumour/ or Gastrointestinal stromal tumour/ or exp Intestine tumour/ or exp Pancreas tumour/ or exp Stomach tumour/)) or (Pancreatic neuroendocrine tumour/ or Pancreas islet cell tumour/ or Glucagonoma/ or Insulino$\mathrm{ma}$ / or Pancreas islet cell carcinoma/ or Gastrinoma/ or Glucagonoma/) or (((gastroenteropancreatic or gastro-enteric pancreatic or gastro-entero-pancreatic or pancreas or pancreatic) and (neuroendocrine and (tumour ${ }^{\star}$ or tumour ${ }^{\star}$ or neoplasm ${ }^{\star}$ or carcinoma*))) or GEPNET or GEP-NET* or GEPNEC* or GEP-NEC*).mp.

Therapy search filter

(dm or dt or su or th or rt).fs. or segmentectomy.mp. or resection.mp. or debulk ${ }^{\star} . \mathrm{mp}$. or cryoablat ${ }^{\star} . \mathrm{mp}$. or cryosurger ${ }^{\star} . \mathrm{mp}$. or radioablat*.mp. or radiofrequency ablat ${ }^{\star}$.mp. or radio-frequency ablat ${ }^{\star} . \mathrm{mp}$. or RFablat*.mp. or thermoablat*.mp. or Cryosurgery/ or Liver resection/ or liver trans- 
plant.mp. or local ablat*.mp. or transarterial embolization.mp. or transarterial embolisation.mp. or transarterial chemoembolization.mp. or transarterial chemoembolisation.mp. or radioembolization.mp. or radioembolisation.mp. or somatostatin.mp. or chemotherapy.mp. or chemotherapies.mp. or peptide receptor radiotherapy.mp. or targeted molecular therapy.mp. or radiopeptide.mp. or DOTATOC.mp. or DOTATATE.mp. or PRRT.mp.

Study design filter (random $^{\star}$ or factorial ${ }^{\star}$ or crossover ${ }^{\star}$ or (cross adj over ${ }^{\star}$ ) or placebo ${ }^{\star}$ or (doubl ${ }^{\star}$ adj blind ${ }^{\star}$ ) or (singl ${ }^{\star}$ adj blind) or assign* or allocat* or volunteer ${ }^{\star}$ ).mp. or Crossover-procedure/ or Double-blind-procedure/ or Single-blind-procedure/ or Randomized-controlled-trial/

WHAT'S NEW

\begin{tabular}{lll}
\hline Date & Event & Description \\
\hline 7 August 2020 & Amended & Edits to author affiliations. \\
\hline
\end{tabular}

\section{HIST O R Y}

Protocol first published: Issue 8, 2020

\section{CONTRIBUTIONS OFAUTHORS}

MAW, LB, MB and RMK designed and drafted the protocol.

All authors approved the final version of the protocol and will do the same with the final manuscript.

\section{DECLARATIONS OF INTEREST}

Martin Alexander Walter: none known

Marko Spanjol: none known

Attila Kollár: Advisory board and meeting honoraria from IPSEN.

Lukas Bütikofer: Affiliation with CTU Bern, University of Bern, which has a staff policy of not accepting honoraria or consultancy fees. However, CTU Bern is involved in design, conduct, or analysis of clinical studies funded by not-for-profit and for-profit organizations. In particular, pharmaceutical and medical device companies provide direct funding to some of these studies. For an up-to-date list of CTU Bern's conflicts of interest see http://www.ctu.unibe.ch/research/declaration_of_interest/index_eng.html.

Viktoria L Gloy: none known

Rebecca Anne Dumont: none known

Christian A Seiler: none known

Emanuel R Christ: Advisory board honoraria from IPSEN, Novartis and Pfizer.

Piotr Radojewski: none known

Matthias Briel: none known

Reto Martin Kaderli: meeting honoraria from IPSEN.

\section{SOURCES OF SUPPORT}

\section{Internal sources}

- None to declare, Other

\section{External sources}

- No sources of support supplied 